\title{
Compositional dependence of optical band gap and refractive index in lead and bismuth borate glasses
}

Saisudha B. Mallur, Tyler Czarnecki, Ashish Adhikari and Panakkattu K. Babu

Department of Physics, Western Illinois University, Macomb, Illinois-61455, USA

\begin{abstract}
We prepared a series of lead and bismuth borate glasses by varying $\mathrm{PbO} / \mathrm{Bi}_{2} \mathrm{O}_{3}$ content and studied refractive index and optical band gap as a function of glass composition. Refractive indices were measured very accurately using a Brewster's angle set up while the optical band gaps were determined by analyzing the optical absorption edge using the Mott-Davis model. Using the Lorentz-Lorentz method and the effective medium theory, we calculated the refractive indices and then compared them with the measured values. Bismuth borate glasses show better agreement between the calculated values of the refractive index and experimental values. We used a differential method based on Mott-Davis model to obtain the type of transition and optical band gap $\left(\mathrm{E}_{\mathrm{opt}}\right)$ which in turn was compared with the value of $\mathrm{E}_{\mathrm{opt}}$ obtained using the extinction coefficient. Our analysis shows that in both lead and bismuth borate glasses, the optical band gap arises due to direct forbidden transition. With increasing $\mathrm{PbO} / \mathrm{Bi}_{2} \mathrm{O}_{3}$ content, the absorption edge shifts toward longer wavelengths and the optical band gap decreases. This behavior can be explained in terms of changes to the $\mathrm{Pb}-\mathrm{O} / \mathrm{Bi}-\mathrm{O}$ chemical bonds with glass composition. We obtained a new empirical relation between the optical band gap and the refractive index which can be used to accurately determine the electronic oxide polarizability in lead and bismuth oxide glasses.
\end{abstract}


KEYWORDS: A. Glasses, B. Optical properties, D. Dielectric properties

\section{Introduction}

Lead and bismuth borate glasses represent two interesting glass forming systems which exhibit a considerable range of variation in optical properties as a function of glass composition [1-7]. The influence of $\mathrm{PbO} / \mathrm{Bi}_{2} \mathrm{O}_{3}$ on the structure of these glasses is unique since they can act as network formers as well as network modifiers [8-11]. The effect is also seen on the glass transition temperature. With the addition of $\mathrm{PbO}$ from $30 \mathrm{~mol} \%$ to $70 \mathrm{~mol} \%$ the glass transition temperature decreases from $500{ }^{\circ} \mathrm{C}$ to $275{ }^{\circ} \mathrm{C}$ [12]. In addition, these glasses have large refractive indices, strong absorption in the ultraviolet region, good radiation shielding properties and wide transmission range which make them suitable as host glasses for lasing rare earth ions. The highly polarizable nature of heavy metal cations is responsible for the high linear refractive index of these glasses which also make them promising materials for use in the field of nonlinear optics. Structural groups like boroxol, pentaborates, and diborates containing three and four coordinated borons, and ring- and chain-type metaborate groups containing nonbridging oxygen (NBO), are found to be present in lead and bismuth borate glasses for different $\mathrm{PbO} / \mathrm{Bi}_{2} \mathrm{O}_{3}$ concentrations which can strongly influence the physical and optical properties [11,13]. Hence studying the optical properties, in particular, the refractive index and optical band gap of lead and bismuth borate glasses and how these properties vary with glass composition is of great interest for practical applications.

In the present work, we investigated the compositional dependence of optical properties of $\mathrm{PbO}-\mathrm{B}_{2} \mathrm{O}_{3}$ and $\mathrm{Bi}_{2} \mathrm{O}_{3}-\mathrm{B}_{2} \mathrm{O}_{3}$ for a broad range of glass compositions. We measured density $(\rho)$, refractive index $(n)$ and optical band gap $(\eta)$ and determined the average electronic oxide polarizability of these glasses. We used Lorentz-Lorenz (LL) method and effective medium 
theory (EMT) to calculate the refractive index $n$ and then the experimental values of the refractive index were compared with the calculated values. Refractive indices calculated using EMT and LL methods show better agreement with the experimental results for the case of bismuth borate glasses than for lead borate glasses.

Optical absorption spectra were analyzed using the Mott-Davis model [14] to determine $\eta$. In order to determine the type of optical transition, we used the relation between the imaginary part of dielectric constant $\left(\varepsilon_{2}\right)$ and extinction coefficient $(\kappa)$. Our analysis shows that in both lead and bismuth borate glasses, the absorption edge arise due to indirect forbidden transitions. $E_{\text {opt }}$ for both glass systems is found to decrease with increasing $\mathrm{PbO} / \mathrm{Bi}_{2} \mathrm{O}_{3}$ content which is explained in terms of the $\mathrm{Pb}-\mathrm{O}$ and $\mathrm{Bi}-\mathrm{O}$ covalency. Finally, for our binary oxide glass systems with different $\mathrm{PbO} / \mathrm{Bi}_{2} \mathrm{O}_{3}$ composition, we obtained an empirical relation between the optical band gap and the refractive index. This empirical relation is capable of accurately reproducing our electronic oxide polarizability calculated from $n$ and $E_{\text {opt }}$

\section{Experimental}

We prepared binary lead borate glasses with the composition $\mathrm{xPbO}:(100-\mathrm{x}) \mathrm{B}_{2} \mathrm{O}_{3}$ with $\mathrm{x}=$ $30-70 \mathrm{~mol} \%$ and binary bismuth borate glasses with the composition $\mathrm{xBi}_{2} \mathrm{O}_{3}$ :(100-x) $\mathrm{B}_{2} \mathrm{O}_{3}$ with $\mathrm{x}$ $=30-60 \mathrm{~mol} \% \mathrm{x}$ was varied in steps of $10 \mathrm{~mol} \%$ giving 5 glass samples for the lead borate system (PbB30-PbB70) and 4 glass samples for the bismuth borate system (BiB30-BiB60), respectively. The raw materials were thoroughly mixed and melted in the range $800-1000{ }^{\circ} \mathrm{C}$. The melt was air quenched by pouring it on a thick brass plate and covering it immediately with another brass plate. The glass samples thus obtained were annealed at $400^{\circ} \mathrm{C}$ for $3 \mathrm{hrs}$ to remove thermal strains. Annealed glass samples were polished using a lapping machine (Inland 
SwapTop) to obtain well reflecting flat surfaces. The sample density was measured by the Archimedes' method using xylene. The refractive index of the glasses is measured by the Brewster angle method using a PASCO set up (OS-8170) with a diode laser as the source. When unpolarized light of $650 \mathrm{~nm}$ wavelength is incident on the surface of the sample at the Brewster angle ( $\theta=\tan ^{-1} n$, where $n$ is the refractive index of the glass) the reflected light is rendered linearly polarized with the electric vector transverse to the plane of incidence. The reflected light passes through a polarizer and is detected by a light sensor. The angle of reflection is measured by a rotary motion sensor mounted on a spectrophotometer table (OS-8539). The intensity of the reflected polarized light versus reflected angle is graphed and a parabolic fit to this data then provides a very accurate determination of the Brewster's angle, which is used to calculate the refractive index of our glass samples [15]. The accuracy of these measurements is around \pm 0.01 . It may be pointed out that the refractive index does not vary significantly with wavelength and, hence, we have measured $n$ at a single wavelength for each sample using a diode laser as the source. Optical absorption spectra of the glasses in the UV-VIS region were recorded at room temperature on polished samples of size $1 \mathrm{~cm} \times 1 \mathrm{~cm} \times 1 \mathrm{~mm}$ using a Varian/Cary 5G spectrometer in the wavelength range $190-1000 \mathrm{~nm}$. The resolution of this spectrometer is $\pm 0.02 \mathrm{~nm}$.

\section{Results and Discussion}

\subsection{Refractive Index}

Dimitrov et al. [4] have reported variation of refractive index of $\mathrm{PbO}: \mathrm{B}_{2} \mathrm{O}_{3}$ glasses with $\mathrm{PbO}$ content varying from $30 \mathrm{~mol} \%$ to $70 \mathrm{~mol} \%$ and Honma et al. [16] for $\mathrm{Bi}_{2} \mathrm{O}_{3}: \mathrm{B}_{2} \mathrm{O}_{3}$ glasses with $\mathrm{Bi}_{2} \mathrm{O}_{3}$ content varying from 30 to $60 \mathrm{~mol} \%$ using ellipsometric measurements. Their refractive index values vary from 1.67 to 2.00 for lead borate glasses and 1.84 to 2.10 for bismuth borate 
glasses with increasing $\mathrm{PbO} / \mathrm{Bi}_{2} \mathrm{O}_{3}$ content. In our binary lead borate glasses and bismuth borate glasses, the refractive indices measured using the Brewster's angle method shows that $n$ varies from 1.66 to 1.92 for lead borate glasses and from 1.92 to 2.21 for bismuth borate glasses. Table 1 lists the experimental refractive indices measured by Brewster's angle method.

Refractive index in glasses is usually expressed by the Lorentz-Lorenz equation [17]

$$
R_{m}=\left(\frac{n^{2}-1}{n^{2}+2}\right) \frac{M}{\rho}=\left(\frac{n^{2}-1}{n^{2}+2}\right) V_{m}
$$

where $R_{\mathrm{m}}$ is the molar refraction, $M$ is the molar mass, $\rho$ is the density, $V_{\mathrm{m}}$ is the molar volume, and $n$ is the refractive index. The molar refraction can be expressed as a function of polarizability $\left(\alpha_{m}\right)$ of a molecule as $[17,18]$ :

$$
\begin{gathered}
R_{m}=\frac{4 \pi \alpha_{m} N_{A}}{3} \\
R_{m}=2.52 \alpha_{m}
\end{gathered}
$$

where $N_{\mathrm{A}}$ is the Avogadro's number and $\alpha_{m}$ is expressed in $\AA^{3}$. In the first approximation, $\alpha_{m}$ and $R_{m}$ can be considered as additive quantities. For an oxide system with chemical formula $A_{p} O_{q}$ it can be expressed by the relation [6]:

$$
R_{m}=p R_{i}+q R_{O^{2-}}=2.52\left(p \alpha_{i}+q \alpha_{O^{2-}}\right)
$$

where $R_{i}$ and $R_{O^{2-}}$ are the ionic refraction and $\alpha_{i}$ and $\alpha_{0^{2-}}$ are the polarizabilities of the cation and the oxide ion, respectively.

Since the molar refraction is an additive quantity, we can assume that for our binary system

$$
R_{m, t}=\sum_{i} f_{i} R_{m, i}
$$


where $R_{m, t}$ is the molar refraction of the binary glass, $f_{\mathrm{i}}$ is molar fraction and $R_{m, i}$ is the molar refraction of the $i$ th oxide. As a first approximation using $\alpha_{i}$ and $\alpha_{0^{2-}}$ reported for simple oxides we calculated $\alpha_{m}$ and $R_{m, t}$ values [6,19]. Then using equation (1), the refractive index by LL method $\left(n_{L L}\right)$ is calculated.

Maxwell-Garnett theory [20] and Bruggeman model [21] are two major EMTs in use today which can be extended to calculate the refractive index of multicomponent mixtures [22]. Maxwell-Garnett theory is quite successful in predicting features of a two-component system when one of the components occupies only a small fraction of the total volume but becomes inaccurate when the two components have comparable volumes. The Bruggeman model is a better EMT for such systems [22]. This model describes the refractive index of a two-component composite material by considering it as an inter-dispersed medium of the two constituent materials. One can apply the geometric parameters of the Bruggeman model to the general expressions for the optical constants of composites to arrive at an explicit prediction for a twocomponent system as:

$$
\frac{\varepsilon_{a}-\varepsilon_{e f f}}{\varepsilon_{a}+2 \varepsilon_{e f f}} V_{a}+\frac{\varepsilon_{b}-\varepsilon_{e f f}}{\varepsilon_{b}+2 \varepsilon_{e f f}} V_{b}=0
$$

where $\varepsilon_{a}$ and $\varepsilon_{b}$ are the dielectric constants and $V_{a}$ and $V_{b}$ are the volume fractions of the two components $a$ and $b$, respectively. $\varepsilon_{\text {eff }}$ is the effective dielectric constant of the mixture. In general, for dielectric constant of a mixture $\quad\left(\varepsilon_{m}\right)$, equation (6) can be written as

$$
\sum_{i}\left(\frac{\varepsilon_{i}-\varepsilon_{m}}{\varepsilon_{i}+2 \varepsilon_{m}}\right) V_{i}=0
$$


where $\varepsilon_{i}=n_{i}^{2}$ is the dielectric constant of the $i$ th component whose volume fraction is $V_{i}$. Using the known values of refractive indices $n(\mathrm{PbO})=2.51, n\left(\mathrm{~B}_{2} \mathrm{O}_{3}\right)=1.64[6] n\left(\mathrm{Bi}_{2} \mathrm{O}_{3}\right)=2.50$, [23], known values of the densities of binary components $\rho(\mathrm{PbO})=9.53 \mathrm{~g} / \mathrm{cm}^{3}, \rho\left(\mathrm{Bi}_{2} \mathrm{O}_{3}\right)=8.90 \mathrm{~g} / \mathrm{cm}^{3}$, $\rho\left(\mathrm{B}_{2} \mathrm{O}_{3}\right)=2.46 \mathrm{~g} / \mathrm{cm}^{3}$ and experimentally measured values of densities of our glasses, we calculated $\varepsilon_{m}$ as a function of glass composition, which was then used to calculate the refractive index.

The experimental refractive index values $\left(n_{\exp }\right)$, the refractive index values calculated according to LL equation ( $\left.n_{L L}\right)$, and according to EMT $\left(n_{E M T}\right)$ are presented in the Table 1 and plotted in Figures 1(a) and 1(b) for lead and bismuth borate glasses, respectively. In lead borate glass, the calculated refractive indices are about 10-15\% larger than the experimental values, whereas in bismuth borate glasses both EMT and LL methods give better overall agreement between calculated and experimental values. To understand the difference between lead borate and bismuth borate glasses, we must examine the details of the structural changes that occur as a function of $\mathrm{PbO} / \mathrm{Bi}_{2} \mathrm{O}_{3}$ concentration. In lead borate glasses, addition of $\mathrm{PbO}$ converts 3coordinated boron $\left(\mathrm{B}_{3}\right)$ to 4 -coordinated boron $\left(\mathrm{B}_{4}\right)$. The fraction of $\mathrm{B}_{4}$ reaches a maximum around 45-50 mol\% of $\mathrm{PbO}$ concentration and with further addition of $\mathrm{PbO}$, back conversion of $\mathrm{B}_{4}$ to $\mathrm{B}_{3}$ takes place with the formation of NBO. For $\mathrm{PbO}$ concentrations above 65 mol\%, most of the oxygen bonded to the boron atoms are NBO [11]. Similar changes are observed in bismuth borate glasses also. Addition of $\mathrm{Bi}_{2} \mathrm{O}_{3}$ to $\mathrm{B}_{2} \mathrm{O}_{3}$, results in the conversion of $\mathrm{B}_{3}$ to $\mathrm{B}_{4}$ with the fraction of $\mathrm{B}_{4}$ reaching a maximum around $40 \mathrm{~mol} \%$ of $\mathrm{Bi}_{2} \mathrm{O}_{3}$. With further addition of $\mathrm{Bi}_{2} \mathrm{O}_{3}$, back conversion of $\mathrm{B}_{4}$ to $\mathrm{B}_{3}$ takes place resulting in the formation of NBO. Thus both lead and bismuth borate glasses show significant changes to the boron coordination with changing glass composition. However, there is a fundamental difference between these two types of glasses. 
Due to the higher polarizability of $\mathrm{Bi}$ atoms, $\mathrm{Bi}_{2} \mathrm{O}_{3}$ can form a glass with a trace of $\mathrm{B}_{2} \mathrm{O}_{3}$ whereas $\mathrm{PbO}$ cannot. It appears that $\mathrm{Bi}_{2} \mathrm{O}_{3}$ can form its own network not needing the presence of borate groups for network interconnection [24] whereas $\mathrm{PbO}$ needs the presence of $\mathrm{B}_{2} \mathrm{O}_{3}$ to form the glass network [11]. Thus in bismuth borate glasses, varying the $\mathrm{Bi}_{2} \mathrm{O}_{3}$ content does not significantly alter the immediate surroundings of the $\mathrm{Bi}_{2} \mathrm{O}_{3}$ molecules as much as it happens with $\mathrm{PbO}$ in lead borate glasses. In the theoretical calculation of the refractive index by both LL and EMT methods, only changes of $R_{m}$ and $V_{m}$ with composition are taken into account whereas changes in the metal ion and oxide ion polarizability are neglected. But from the discussion above, it is clear that $\mathrm{PbO}$ undergoes a larger alteration to its immediate chemical environment compared to $\mathrm{Bi}_{2} \mathrm{O}_{3}$ when the glass composition is varied. Therefore, the assumption that the average cation electronic polrazability is a constant for $\mathrm{PbO}$ is likely to be inaccurate resulting in the difference between the experimental and calculated values of $n$ for $\mathrm{PbO}-\mathrm{B}_{2} \mathrm{O}_{3}$ glasses.
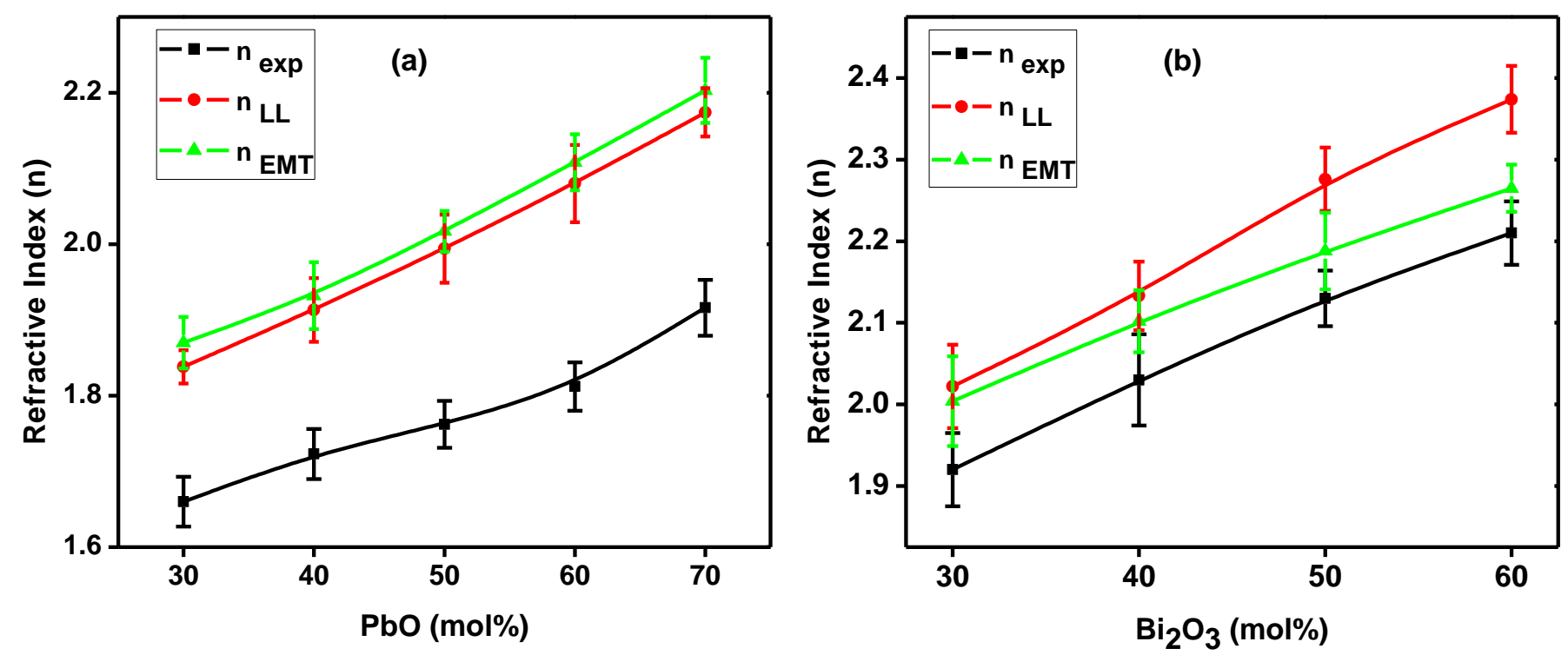

Figure 1. Variation of $n_{\exp } n_{L L}, n_{E M T}$ of (a) lead borate glasses with $\mathrm{PbO}$ content (b) bismuth borate glasses with $\mathrm{Bi}_{2} \mathrm{O}_{3}$ content. 
Table 1. Glass composition, density $(\rho)$, molar volume $\left(V_{m}\right)$, experimental refractive index $\left(n_{\text {exp }}\right)$, refractive index by Lorentz-Lorenz method $\left(n_{L L}\right)$, refractive index by effective medium theory $\left(n_{E M T}\right)$ of lead and bismuth borate glasses

\begin{tabular}{|c|c|c|c|c|c|}
\hline \multirow[t]{2}{*}{$\begin{array}{l}\text { Glass } \\
\text { Composition }\end{array}$} & \multicolumn{5}{|c|}{$\begin{array}{l}\text { Physical } \\
\text { Parameters }\end{array}$} \\
\hline & $\rho\left(\mathrm{g} / \mathrm{cm}^{3}\right)$ & $V_{m}\left(\mathrm{~cm}^{3} / \mathrm{mol}\right)$ & $\overline{n_{\text {exp }}}$ & $n_{L L}$ & $n_{E M T}$ \\
\hline PbB30 & $4.232 \pm 0.004$ & $27.34 \pm 0.11$ & $1.66 \pm 0.03$ & $1.84 \pm 0.02$ & $1.87 \pm 0.03$ \\
\hline PbB40 & $4.589 \pm 0.002$ & $28.56 \pm 0.06$ & $1.72 \pm 0.03$ & $1.91 \pm 0.04$ & $1.93 \pm 0.04$ \\
\hline PbB50 & $5.068 \pm 0.006$ & $28.89 \pm 0.2$ & $1.76 \pm 0.03$ & $1.99 \pm 0.05$ & $2.02 \pm 0.03$ \\
\hline PbB60 & $5.519 \pm 0.005$ & $29.31 \pm 0.2$ & $1.81 \pm 0.03$ & $2.08 \pm 0.05$ & $2.11 \pm 0.04$ \\
\hline PbB70 & $6.032 \pm 0.007$ & $29.36 \pm 0.3$ & $1.92 \pm 0.04$ & $2.17 \pm 0.03$ & $2.20 \pm 0.04$ \\
\hline BiB30 & $5.511 \pm 0.008$ & $34.21 \pm 0.3$ & $1.922 \pm 0.05$ & $2.02 \pm 0.05$ & $2.00 \pm 0.06$ \\
\hline BiB40 & $6.260 \pm 0.003$ & $36.45 \pm 0.12$ & $2.03 \pm 0.06$ & $2.13 \pm 0.04$ & $2.10 \pm 0.04$ \\
\hline BiB50 & $7.039 \pm 0.005$ & $38.04 \pm 0.2$ & $2.13 \pm 0.03$ & $2.28 \pm 0.04$ & $2.19 \pm 0.05$ \\
\hline BiB60 & $7.609 \pm 0.007$ & $40.40 \pm 0.3$ & $2.21 \pm 0.04$ & $2.37 \pm 0.04$ & $2.27 \pm 0.03$ \\
\hline
\end{tabular}




\subsection{The optical band gap}

The study of optical absorption in the ultraviolet region is a useful technique to understand the electronic band structures of glasses. In the optical absorption spectrum of glasses, one can observe a rapid rise in the absorbance toward lower wavelengths. The rapid rise of absorption coefficient is referred to as the fundamental absorption edge (UV "cut off"). Beyond this UV “cut off” value, glasses will be opaque to electromagnetic radiation. Most of the oxide glasses are opaque at wavelengths shorter than $200 \mathrm{~nm}$ when the photon energy becomes greater than the optical band gap that exists between the valence and conduction bands. In glasses, measuring this band gap can provide information regarding structural alterations and the nature of chemical bonds in the matrix. The width of the localized states in the band gap which arises due to the disorder in the matrix can also be determined from the analysis of the optical absorption spectra.

Basically, two types of transitions can occur at the fundamental absorption edge of both crystalline and non-crystalline materials - direct transitions and indirect transitions. For the direct optical transitions, it is essential that the wave vector for an electron remains the same as it absorbs a photon. On the other hand, the indirect transitions will also involve simultaneous interaction between the electron and lattice vibrations (phonons). In this case, the wave vector of the electron can change in an optical transition due to the absorption/emission of a phonon. In other words for indirect transitions, the minimum of the conduction band lies in a different kspace direction compared to the maximum of the valence band.

The optical absorption edge of binary lead borate and bismuth borate glasses is shown in Figures 2(a) and 2(b), respectively. As the concentration of $\mathrm{PbO} / \mathrm{Bi}_{2} \mathrm{O}_{3}$ increases, the position of 
the fundamental absorption edge shifts from 344 to $458 \mathrm{~nm}$ in lead borate glasses and from 400 to $460 \mathrm{~nm}$ in bismuth borate glasses. For comparison, in lead silicate glasses the absorption edge was found to shift from 340 to $400 \mathrm{~nm}$ with increasing PbO content [25]. The absorption edge of these glasses is not as sharp as in crystalline compounds due to the disorder in the matrix. The shifts in the optical absorption edge to longer wavelengths indicate that the $E_{\text {opt }}$ values decrease with increase in $\mathrm{PbO} / \mathrm{Bi}_{2} \mathrm{O}_{3}$ content which is explained below.

Analyzing the behavior of the optical absorption coefficient $\alpha$, near the fundamental absorption edge is a standard method for investigating optically induced electronic transitions in many materials. We can obtain experimental optical band gap directly by using relation between absorption coefficient and extinction coefficient which is given by [26]

$$
\kappa=\frac{\alpha \lambda}{4 \pi}
$$

The extinction coefficient is plotted as a function of energy hv (Figs 3(a) and 3(b)). By extrapolating the linear region of the extinction coefficient to zero, we obtained the experimental optical band gaps for different glass compositions as listed in Table 2.

Mott and Davis [14] suggested the following form for the absorption coefficient $\alpha(v)$ as a function of photon energy

$$
\alpha(v)=B\left(h v-E_{o p t}\right)^{m} / h v
$$

Here, for direct transitions we have $m=1 / 2$ or $3 / 2$ depending on whether the transition is allowed or forbidden and for indirect transitions, $m=2$ or 3 depending on whether the transition is allowed or forbidden, $B$ is a constant, and $E_{\text {opt }}$ is the optical band gap. The type of transition can 
be obtained from the value of $m$. In order to determine the unique value of $m$, we followed the differential method [27]. We rewrite equation (10) as follows.

$$
\frac{d[\ln (\alpha h v)}{d[h v]}=\frac{m}{h v-E_{o p t}}
$$

The differential curve $\frac{d[\ln (\alpha h v)}{d[h v]}$ versus $h v$ has a discontinuity at a particular energy value ( $h v$ $\left.=\mathrm{E}_{\mathrm{opt}}\right)$ which gives the optical band gap $E_{\text {opt.. }}$ In order to find the type of transition, the $E_{\text {opt }}$ values are calculated by extrapolation of the linear parts of $(\alpha h v)^{1 / m}$ vs. hv curves to $(\alpha h v)^{1 / m}=0$ for different values of $m$ (Table 2). By comparing the value from the differential curve with the optical band gap obtained using different values of $m$, we find good agreement with $E_{\text {opt }}$ values for $m=3 / 2$. Hence we can conclude that for lead and bismuth borate glasses the optical band gap arises due to the direct forbidden transition. Thus the experimental optical band gap obtained directly from the extinction coefficient matches well with the Mott-Davies relation for $m=3 / 2$ transition. Figures 4 (a) and 4(b) show the plot of $(\alpha \mathrm{h} v)^{2 / 3}$ vs. hv for various $\mathrm{PbO} / \mathrm{Bi}_{2} \mathrm{O}_{3}$ contents. $E_{\text {opt }}$ is found to decrease from 3.52 to $2.74 \mathrm{eV}$ for lead borate glasses and from 3.10 to $2.78 \mathrm{eV}$ for bismuth borate glasses with increasing concentration of the metal oxide as shown in Figures 5(a) and 5(b). In lead borate glasses, there is a slope change in $E_{o p t}$ vs. composition curve around $50 \mathrm{~mol} \%$ of $\mathrm{PbO}$ indicating a significant electronic alteration around that composition, whereas a similar change occurs in bismuth borate glasses around 30-40 mol\% of $\mathrm{Bi}_{2} \mathrm{O}_{3}$.

The origin of optical band gap in lead silicate glasses is well established [28]. According to this work, $E_{\text {opt }}$ of binary lead borate glasses represents the excitation energy of electrons from the hybridized orbitals of $6 \mathrm{~s}$ lead and $2 \mathrm{p}$ non-bridging oxygens states to the conduction band (6p states of lead forms the conduction band). The $E_{\text {opt }}$ depends on the energy level of the upper 
valence band edge which is determined by the separation of $6 \mathrm{~s}$ lead and $2 \mathrm{p}$ oxygen states. A theoretical study of the energy bands based on ionic model, indicates that the separation between 6s lead and $2 p$ oxygen states is very sensitive to the charge on the oxygen ion [29]. As the ionicity of the oxygen decreases, the energy of the 6 s lead state decreases and the $2 p$ oxygen state increases, so the hybridization of these two states increases. This implies that an increase in the covalent character of the $\mathrm{Pb}-\mathrm{O}$ bond decreases the optical band gap. Such an increase in covalency of $\mathrm{Pb}-\mathrm{O}$ bond with $\mathrm{PbO}$ content in lead borate glasses has been suggested by far infrared spectrum [30]. From NMR results $[13,31]$, it is found that the chemical shift of $B_{3}$ units changes with $\mathrm{PbO}$ concentration. This change shows that the $\mathrm{PbO}$ units which are formed due to the addition of $\mathrm{PbO}$ to $\mathrm{B}_{2} \mathrm{O}_{3}$, bridge preferentially to $\mathrm{B}_{3}$ units. This results in the formation of $\mathrm{Pb}$ $\mathrm{O}-\mathrm{B}$ linages, which arise as a result of the increasing covalency of the $\mathrm{Pb}-\mathrm{O}$ bond. This increase in covalency of the $\mathrm{PbO}$ bond is a consequence of the high polarizability of $\mathrm{Pb}$ ions and the directional nature of the $\mathrm{PbO}$ bond which effectively reduces the optical band gap. In bismuth borate glasses the reduction of $E_{\text {opt }}$ with $\mathrm{Bi}_{2} \mathrm{O}_{3}$ content is also due to an increase in the covalent nature of $\mathrm{Bi}-\mathrm{O}$ bond. The covalency of $\mathrm{Bi}-\mathrm{O}$ bond is larger compared to $\mathrm{Pb}-\mathrm{O}$ bond due to the highly polarizable and directional nature of $\mathrm{Bi}-\mathrm{O}$ bond. Hence, the $E_{\text {opt }}$ values in bismuth borate glasses are smaller compared to their lead borate counterparts. .

The optical absorption edge of many amorphous semiconductors is characterized by the tail of the absorption edge where the absorption coefficient $\alpha(v)$ rises exponentially with photon energy hv as

$$
\alpha(v)=A \exp \left(\frac{h v}{\Delta E}\right)
$$


$\Delta E$ (Urbach energy) represents the width of the band tails of localized states in the band gap. The values of $\Delta E$ are calculated from the slopes of the linear plots of $\ln \alpha \mathrm{vs}$. hv in the lower photon energy region and are listed in Table 2 for both lead borate and bismuth borate glasses. The values of $\Delta E$ vary from 0.29 to $0.21 \mathrm{eV}$ for lead borate glasses and 0.23 to $0.19 \mathrm{eV}$ for bismuth borate glasses. Large $\Delta E$ values for these glasses are due to the disorder and the wide distribution of local electric field intensities in the glass matrix compared to the crystalline systems where $\Delta E \sim 0.046-0.066 \mathrm{eV}$ as reported by Mott and Davis [14].
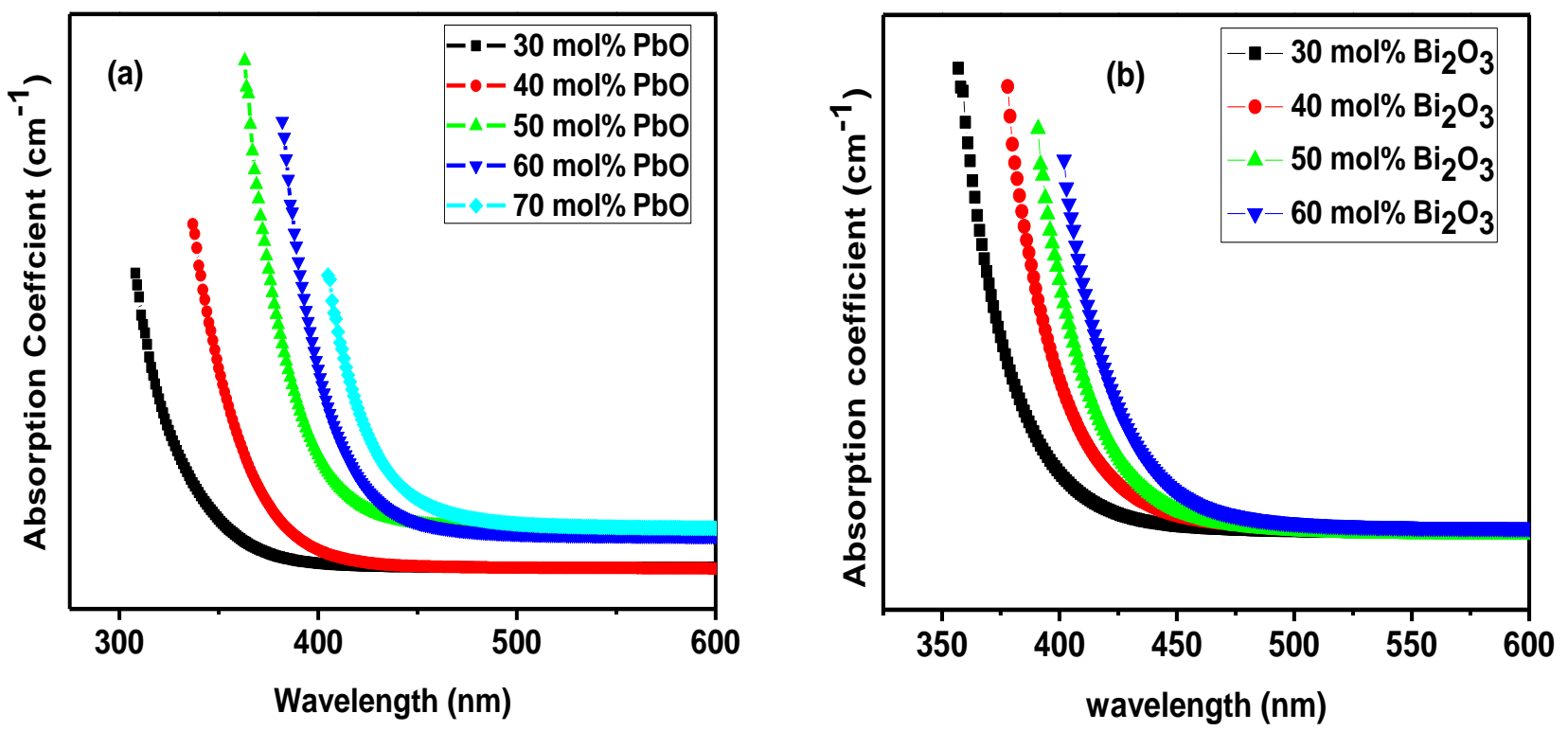

Figure 2. Optical absorption edge of (a) lead borate glasses (b) bismuth borate glasses 

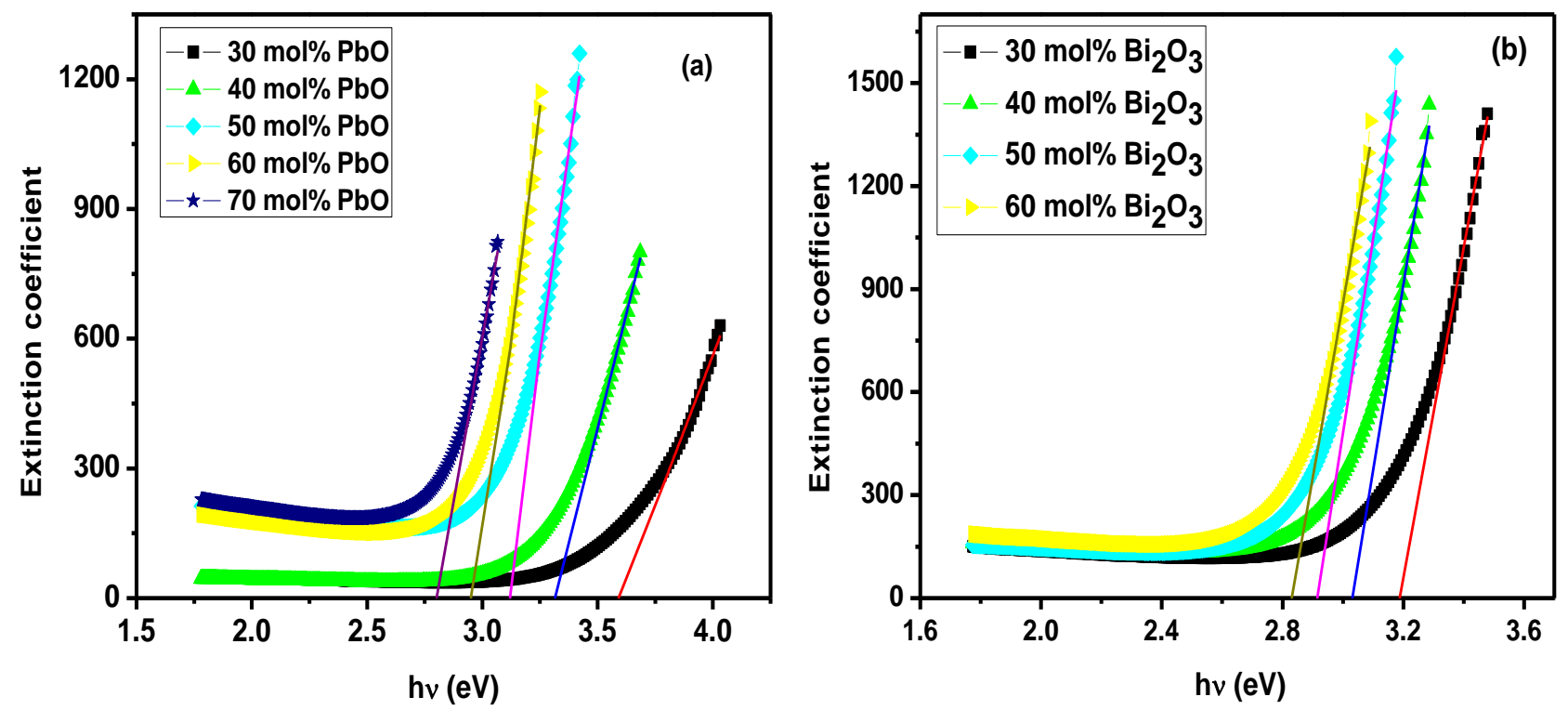

Figure 3. Plot of extinction coefficient vs. hv for (a) lead borate glasses (b) bismuth borate glasses
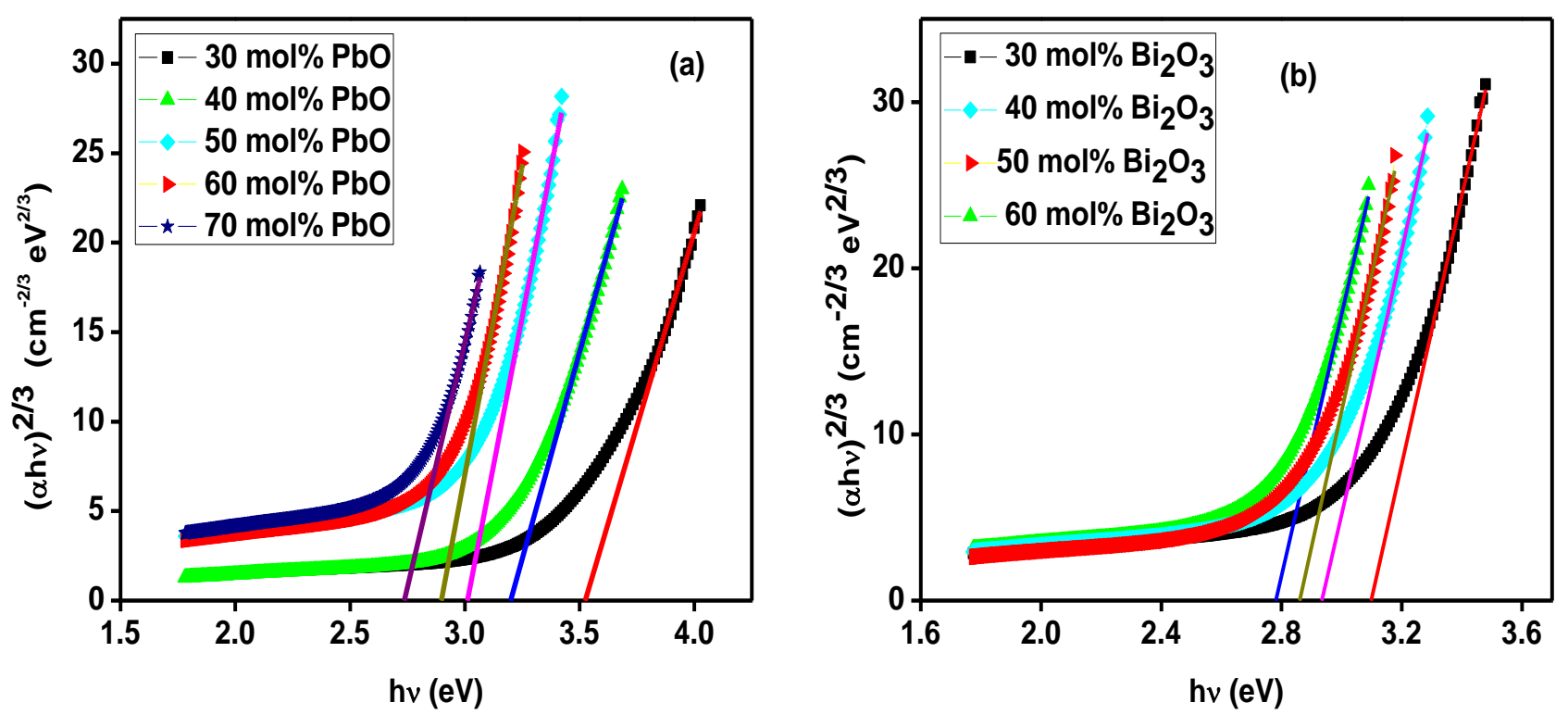

Figure 4. Plot of $(\alpha h v)^{2 / 3}$ vs. hv for (a) lead borate glasses (b) bismuth borate glasses 
Table 2. Variation of $E_{\text {opt }}$ for lead and bismuth borate glasses with glass composition

\begin{tabular}{|c|c|c|c|c|c|}
\hline \multirow[t]{2}{*}{$\begin{array}{c}\text { Optical Bandgap } \\
E_{\text {opt }}(\mathrm{eV}) \\
\end{array}$} & \multicolumn{4}{|c|}{$\begin{array}{c}\text { Glass } \\
\text { Composition } \\
\end{array}$} & \multirow[b]{2}{*}{ PbB70 } \\
\hline & PbB30 & PbB40 & PbB50 & PbB60 & \\
\hline $\begin{array}{l}\mathrm{E}_{\text {opt }} \text { from } k \\
\text { (Experimental) }\end{array}$ & $3.59 \pm 0.04$ & $3.30 \pm 0.05$ & $3.12 \pm 0.04$ & $2.95 \pm 0.05$ & $2.80 \pm 0.03$ \\
\hline $\begin{array}{l}E_{\text {opt }} \text { from } \\
\text { Differential Curve }\end{array}$ & $3.50 \pm 0.05$ & $3.25 \pm 0.04$ & $3.05 \pm 0.03$ & $2.89 \pm 0.04$ & $2.76 \pm 0.04$ \\
\hline $\begin{array}{l}\text { Direct allowed } \\
\text { transition } m=1 / 2\end{array}$ & $3.83 \pm 0.03$ & $3.50 \pm 0.04$ & $3.28 \pm 0.03$ & $3.13 \pm 0.04$ & $2.95 \pm 0.05$ \\
\hline $\begin{array}{l}\text { Direct forbidden } \\
\text { transition } m=3 / 2\end{array}$ & $3.52 \pm 0.03$ & $3.20 \pm 0.04$ & $3.01 \pm 0.04$ & $2.90 \pm 0.03$ & $2.74 \pm 0.04$ \\
\hline $\begin{array}{l}\text { Indirect allowed } \\
\text { transition } m=2\end{array}$ & $3.36 \pm 0.03$ & $3.03 \pm 0.04$ & $2.90 \pm 0.03$ & $2.81 \pm 0.04$ & $2.63 \pm 0.04$ \\
\hline $\begin{array}{l}\text { Indirect forbidden } \\
\text { transition } m=3\end{array}$ & $3.11 \pm 0.03$ & $2.79 \pm 0.04$ & $2.72 \pm 0.04$ & $2.59 \pm 0.04$ & $2.46 \pm 0.03$ \\
\hline \multirow[t]{2}{*}{ Urbach energy $\Delta E$} & $0.291 \pm 0.002$ & $0.233 \pm 0.003$ & $0.232 \pm 0.004$ & $0.204 \pm 0.002$ & $0.213 \pm 0.001$ \\
\hline & BiB30 & BiB40 & BiB50 & BiB60 & \\
\hline $\begin{array}{l}\mathrm{E}_{\mathrm{opt}} \text { from } k \\
\text { (Experimental) }\end{array}$ & $3.19 \pm 0.03$ & $3.03 \pm 0.03$ & $2.91 \pm 0.03$ & $2.83 \pm 0.04$ & \\
\hline $\begin{array}{l}E_{\text {opt }} \text { from } \\
\text { Differential Curve }\end{array}$ & $3.07 \pm 0.04$ & $2.91 \pm 0.05$ & $2.84 \pm 0.04$ & $2.77 \pm 0.02$ & \\
\hline $\begin{array}{l}\text { Direct allowed } \\
\text { transition } m=1 / 2\end{array}$ & $3.33 \pm 0.04$ & $3.16 \pm 0.03$ & $3.06 \pm 0.04$ & $2.98 \pm 0.05$ & \\
\hline $\begin{array}{l}\text { Direct forbidden } \\
\text { transition } m=3 / 2\end{array}$ & $3.10 \pm 0.03$ & $2.93 \pm 0.05$ & $2.86 \pm 0.04$ & $2.78 \pm 0.03$ & \\
\hline $\begin{array}{l}\text { Indirect allowed } \\
\text { transition } m=2\end{array}$ & $3.01 \pm 0.04$ & $2.82 \pm 0.04$ & $2.75 \pm 0.03$ & $2.70 \pm 0.03$ & \\
\hline $\begin{array}{l}\text { Indirect forbidden } \\
\text { transition } m=3\end{array}$ & $2.76 \pm 0.03$ & $2.64 \pm 0.04$ & $2.55 \pm 0.03$ & $2.51 \pm 0.02$ & \\
\hline Urbach energy $\Delta E$ & $0.230 \pm 0.003$ & $0.201 \pm 0.004$ & $0.191 \pm 0.004$ & $0.193 \pm 0.00$ & \\
\hline
\end{tabular}



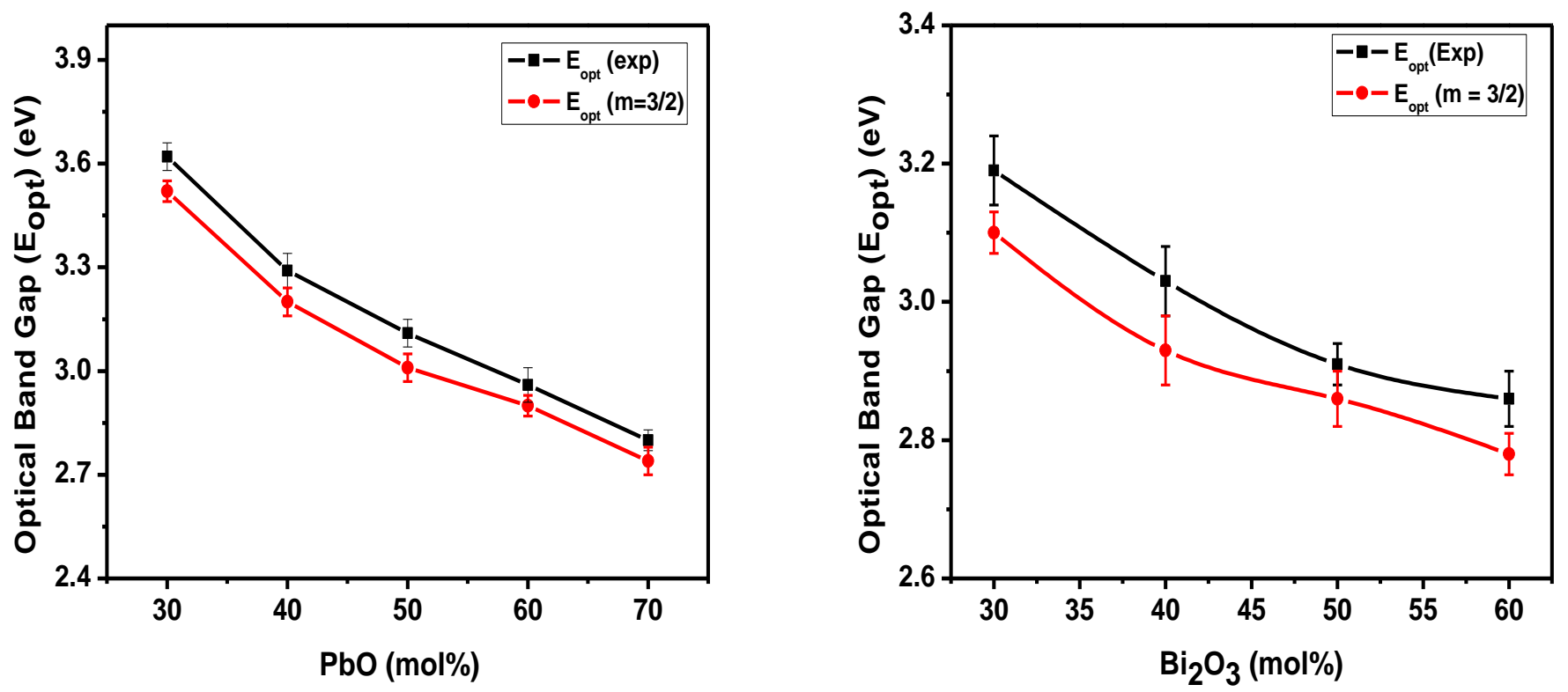

Figure 5. Compositional dependence of experimental optical band gap and optical band gap from Mott-Davis Model for $\mathrm{m}=3 / 2$ transition (a) lead borate glasses (b) bismuth borate glasses

\subsection{Optical band gap and refractive index based electronic polarizability of the oxide ion}

It is known that the nonlinear optical response of a material when exposed to intense light is closely related to its electronic polarization. The macroscopic polarization itself is governed by the electronic polarizability of its individual components. Assuming that molar refractivity $\left(R_{m}\right)$ and polarizability $\left(\alpha_{m}\right)$ are additive quantities, Dimitrov and Sakka [6] obtained the relationship given by equation (4). Combining this relationship with Lorentz-Lorenz equation (1) leads to

$$
\alpha_{O^{2-}}(n)=\left[\left(\frac{V_{m}}{2.52}\right)\left(\frac{n^{2}-1}{n^{2}+2}\right)-\sum_{i} p \alpha_{i}\right] q^{-1}
$$


Where $\left(\frac{n^{2}-1}{n^{2}+2}\right)=\frac{R_{m}}{V_{m}}$ from equation (1)

For simple oxides, Dimitrov and Sakka used the following linear relationship suggested by Duffy $[6,32]$

$$
E_{\text {opt }}=20\left(1-R_{m} / V_{m}\right)^{2}
$$

Vithal et al [33] extended their work to simple binary oxides. They found an empirical relation very close to Duffy's relation which is given as

$$
\sqrt{E_{o p t}}=1.23\left(1-R_{m} / V_{m}\right)+0.98
$$

Plotting $E_{\text {opt }}$ vs. $\left(1-R_{m} / V_{m}\right)$ gave us the following empirical relations

$$
\begin{aligned}
& E_{o p t}=6.73\left(1-R_{m} / V_{m}\right)^{1.46} \\
& E_{o p t}=4.4\left(1-R_{m} / V_{m}\right)^{0.56}
\end{aligned}
$$

for lead borate glasses with $\mathrm{PbO}$ content (Figure 6a) and for bismuth borate glasses with $\mathrm{Bi}_{2} \mathrm{O}_{3}$ content (Figure 6b), respectively. Thus we obtained two new empirical relations between $E_{\text {opt }}$ and $\left(1-R_{m} / V_{m}\right)$. These relations explain the compositional dependence of $E_{\text {opt }}$ for lead and bismuth borate glasses.

Substituting equations (14) and (15) in place of $\left(\frac{n^{2}-1}{n^{2}+2}\right)$ in equation (12) we get for lead borate glasses

$$
\alpha_{O^{2-}}\left(E_{o p t}\right)=\left[\left(\frac{V_{m}}{2.52}\right)\left(1-\left(\frac{E_{o p t}}{6.73}\right)^{0.68}\right)-\sum_{i} p \alpha_{i}\right] q^{-1}
$$

and for bismuth borate glasses, 


$$
\alpha_{O^{2-}}\left(E_{o p t}\right)=\left[\left(\frac{V_{m}}{2.52}\right)\left(1-\left(\frac{E_{o p t}}{4.4}\right)^{1.79}\right)-\sum_{i} p \alpha_{i}\right] q^{-1}
$$

Using the relations (16) and (17), the average electronic oxide polarizability can be calculated from the optical band gap $E_{o p t}$, molar volume $V_{m}$ and cation polarizability $\alpha_{i}$. We also calculated $\alpha_{O^{2-}}$ from $n$ values using equation (12). Results of these two independent calculations are given in Table 3. Duffy's empirical relation is obeyed by a large number of simple oxides. However, for our binary oxide glass systems with different $\mathrm{PbO} / \mathrm{Bi}_{2} \mathrm{O}_{3}$ composition, we find that $\alpha_{O^{2-}}$ calculated using our empirical relations (15) and (16) match better with $\alpha_{O^{2-}}$ obtained from the refractive index values.
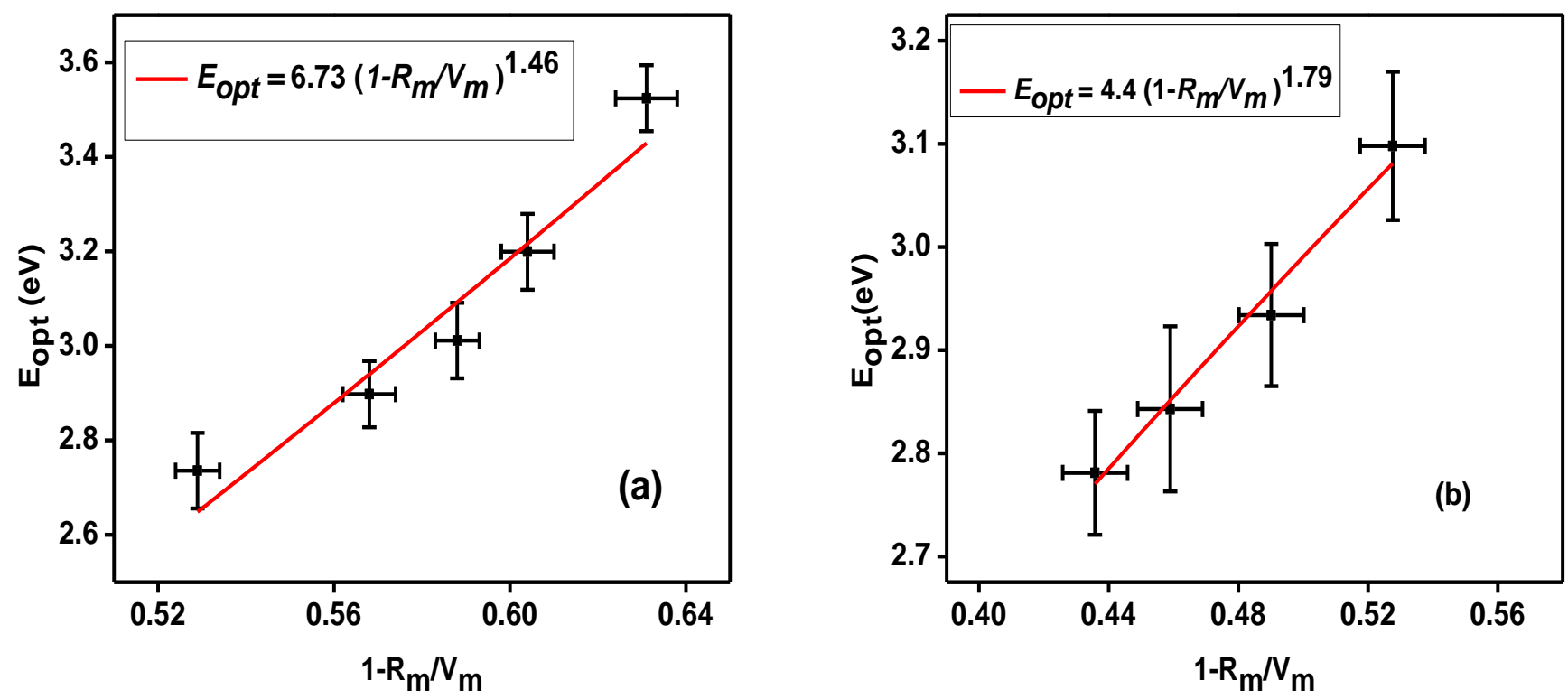

Figure 6. Variation of $E_{o p t}$ with $1-R_{m} / V_{m}$ (a) in lead borate glasses (b) in bismuth borate glasses 
Table 3. Glass composition, molar refraction, molecular polarizability $\left(\alpha_{m}\right)$, electronic oxide polarizability $\left(\alpha_{O^{2-}}\right)(n)$, electronic oxide polarizability $\left(\alpha_{O^{2-}}\right)\left(E_{o p t}\right)$ of lead borate and bismuth borate glasses

\begin{tabular}{|c|c|c|c|c|c|}
\hline $\begin{array}{c}\text { Glass } \\
\text { Composition }\end{array}$ & & & $\begin{array}{c}\text { Physical } \\
\text { Parameters }\end{array}$ & & \\
\hline & $R_{m}$ & $1-R_{m} / V_{m}$ & $\begin{array}{c}\alpha_{m} \\
\left(10^{-24} \mathrm{~cm}^{3}\right)\end{array}$ & $\begin{array}{c}\left(\alpha_{O^{2^{-}}}\right)(n) \\
\left(10^{-24} \mathrm{~cm}^{3}\right)\end{array}$ & $\begin{array}{c}\left(\alpha_{o^{2-}}\right)\left(E_{\text {opt }}\right) \\
\left(10^{-24} \mathrm{~cm}^{3}\right)\end{array}$ \\
\hline $\mathrm{PbB} 30$ & $10.09 \pm 0.1$ & $0.631 \pm 0.007$ & 4.001 & 1.215 & 1.155 \\
\hline $\mathrm{PbB} 40$ & $11.32 \pm 0.2$ & $0.604 \pm 0.006$ & 4.492 & 1.382 & 1.385 \\
\hline $\mathrm{PbB} 50$ & $11.91 \pm 0.12$ & $0.588 \pm 0.005$ & 4.726 & 1.458 & 1.508 \\
\hline $\mathrm{PbB} 60$ & $12.67 \pm 0.18$ & $0.568 \pm 0.006$ & 5.028 & 1.585 & 1.610 \\
\hline $\mathrm{PbB} 70$ & $13.83 \pm 0.15$ & $0.529 \pm 0.005$ & 5.488 & 1.846 & 1.749 \\
\hline BiB30 & $16.16 \pm 0.15$ & $0.528 \pm 0.008$ & 6.413 & 1.835 & 1.806 \\
\hline BiB40 & $18.58 \pm 0.21$ & $0.491 \pm 0.007$ & 7.374 & 2.055 & 2.083 \\
\hline BiB50 & $20.58 \pm 0.19$ & $0.459 \pm 0.009$ & 8.168 & 2.219 & 2.225 \\
\hline BiB60 & $22.80 \pm 0.16$ & $0.436 \pm 0.006$ & 9.046 & 2.412 & 2.388 \\
\hline
\end{tabular}




\section{Conclusions}

Using an accurate method to measure the Brewster's angle, we investigated the compositional dependence of refractive index for $\mathrm{PbO}-\mathrm{B}_{2} \mathrm{O}_{3}$ and $\mathrm{Bi}_{2} \mathrm{O}_{3}-\mathrm{B}_{2} \mathrm{O}_{3}$ glasses for a broad range of glass compositions. We also calculated the refractive index by two theoretical methods (LorentzLorenz and Bruggeman's effective medium theory). We found that in lead borate glasses, the calculated refractive indices are larger than the experimental values, whereas in bismuth borate glasses both EMT and LL methods give better overall agreement between calculated and experimental values. In lead borate glasses $\mathrm{PbO}$ undergoes a larger alteration to its immediate chemical environment compared to $\mathrm{Bi}_{2} \mathrm{O}_{3}$ when the glass composition is varied. Therefore, assuming constant values for the average cation electronic polrazability of $\mathrm{PbO}$ is likely to be incorrect which leads to the difference between the experimental and calculated values of $\eta$ for $\mathrm{PbO}-\mathrm{B}_{2} \mathrm{O}_{3}$ glasses. With increasing $\mathrm{PbO} / \mathrm{Bi}_{2} \mathrm{O}_{3}$ content, the optical absorption edge shifts to longer wavelengths indicating significant changes to the electronic band structure of these glasses. We obtained optical band gap for our systems using Mott-Davis model from the optical absorption edge measurements. In order to find the type of transition, optical band gap is obtained by the differential method using Mott-Davis model and compared with the experimentally obtained value. We found that in both lead borate and bismuth borate glasses, the absorption edge arises due to direct forbidden transitions $(\mathrm{m}=3 / 2)$. The $E_{\text {opt }}$ is found to decrease from 3.52 to $2.74 \mathrm{eV}$ for lead borate glasses and from 3.10 to $2.78 \mathrm{eV}$ in bismuth borate glasses with increasing concentration of the metal oxide. The reduction of $E_{\text {opt }}$ with $\mathrm{PbO} / \mathrm{Bi}_{2} \mathrm{O}_{3}$ content is due to the increase in the covalent nature of $\mathrm{Pb}-\mathrm{O} / \mathrm{Bi}-\mathrm{O}$ bond. The electronic oxide polarizability in lead borate glasses and bismuth borate glasses were calculated on the basis of two different 
properties, linear refractive index $(n)$ and the optical band gap $\left(E_{\text {opt }}\right)$ by using a new empirical relation between $E_{\text {opt }}$ and $n$. Both values show very good agreement.

Acknowledgement

The authors would like to acknowledge a start-up grant from the University Research Council, Western Illinois University. Optical absorption measurements were carried out at the Frederick Seitz Materials Research Laboratory Central Facilities, University of Illinois, which are partially supported by the U.S. Department of Energy under grants DE-FG02-07-ER46453 and DE-FG02-07-ER46471.

\section{References}

[1] I. Opera, H. Hesse, and K. Betzler, Opt. Mater., 26 (2004) 235-237.

[2] X. Zhao, X. Wang, H. Lin, and Z. Wang, Physica B, 390 (2007) 293-300.

[3] O. Sanz, E. Haro-Poniatowski, J. Gonzalo, \& J. M. Fernández Navarro, J. Non-Cryst. Solids, 352 (2006) 761-768.

[4] V.V. Dimitrov, S. Kim, T. Yoko and S. Sakka, J. Ceramic. Soc. Japan, 101 (1993) 59-63

[5] V.V. Dimitrov and S. Sakka., J. Appl. Phys. 79 (1996) 1741-1745

[6] V. Dimitrov, S. Sakka, J. Appl. Phys. 79 (1996) 1736-1740

[7] Joanna Pisarska, Opt. Mater.31(2009)1784-1786

[8] M.B. Saisudha and J. Ramakrishna, Phys. Rev. B., 53 (1996) 6186-6196

[9] M.B. Saisudha, K.S.R. Koteshwara Rao, H.L. Bhat and J. Ramakrishna, J. Appl. Phys. 80, (1996) $4845-4853$

[10] M.B. Saisudha and J.Ramakrishna, Optical Materials, 18 (2002) 403-417

[11] B.N. Meera, A.K.Sood, N. Chandra Bhas, and J. Ramakrishna, J. Non-Cryst. Solids 126, (1990) 224-230 
[12] I. Avramov, Ts. Vassilev, I. Penkov., J. Non-Cryst. Solids, 351 (2005) 472-476

[13] P. J. Bray, M. Leventhal, and H. O. Hooper, Phys. Chem. Glasses 4 (1963) 47-66

[14] N.F. Mott and E.A. Davis, "Electronic processes in non-crystalline materials", $2^{\text {nd }}$ edn (Oxford University Press, Oxford, 1977)

[15] Cristian Bahrim and Wei-Tai Hsu, Am. J. Phys. 77 (2009) 337-343

[16] T. Honma, Y. Benino, T. Komatsu, R. Sato, V. Dimitrov, Phys. Chem. Glasses, 43 (2002) $32-40$

[17] E. A. Moelwyn-Hughes, Physical Chemistry, Pergamon, London 1961

[18] H. Rawson, Properties and Applications of Glass, Elsevier, Amsterdam 1980.

[19] V. Dimitrov, T. Komatsu, J. Solid State Chemistry 163 (2002) 100-112

[20] C. Maxwell-Garnet, Philos. Trans. R. Soc. Cand. 203 (1904) 385, ibid 205 (1906) 237

[21] D. A. G. Bruggeman, Ann. Phys. (Leipzig) 24 (1935) 636-679

[22] R.J. Gehr, G.L. Fischer, R.W. Boyd, J. Opt. Soc. Am. B, 14 (1997) 2310-2314

[23] P. B. Clapham, Br. J. Appl. Phys. 18 (1967) 363-366

[24] A. Vegas, F.H. Cano and S. Garcia-Blanco, J. Solid St. Chem., 17 (1976) 151-155

[25] B.M. Cohen, D.R. Ulmann and R.R. Shaw., J. Non-Cryst.Solids, 12 (1973) 177-188

[26] Seung-Gu Lim, Stas Kriventsov, and Thomas N. Jackson et al, J. Appl.Phys., 91 (2002) $4500-4505$

[27] A. Masuno, H. Inoue, J. Yu, Y. Arai., J. Appl.Phys 108 (2010) 063520-1

[28] I.S. Reshetnikova and V. N. Shapovalov., Optica and Spectroscopy, 75 (1993) 749-752

[29] A.H. Kahn and A.J. Leyendecker., Phys. Rev A., 135 (1964) 1321-1325

[30] P.Tarte and M.J.Portier., The structure of non-crystalline materials, P.H.Gaskell (Ed.) (1977) 227. 
[31] M. Leventhal and P.J. Bray., Phys.Chem.Glasses, 6 (1965) 113-125

[32] J. A. Duffy, J. Solid State Chem., 62 (1986) 145-157

[33] M. Vithal, P. Nachimuthu, T. Banu, and R. Jagannathan., J. Appl. Phys. 81 (1997) 79227926 